\title{
Goal-Directed Fluid Therapy Based on Pulse- Pressure Variation Compared with Standard Fluid Therapy in Patients Undergoing Complex Spine Surgery: A Randomized Controlled Trial
}

\author{
Karuna Wongtangman ${ }^{1}$, Sirichai Wilartratsami ${ }^{2}$, Nattachai Hemtanon ${ }^{1}$, \\ Supinya Tiviraj ${ }^{1}$, Manee Raksakietisak ${ }^{1}$ \\ ${ }^{1}$ Department of Anesthesiology, Faculty of Medicine, Siriraj Hospital, Mahidol University, Bangkok, Thailand \\ ${ }^{2}$ Department of Orthopaedic Surgery, Faculty of Medicine, Siriraj Hospital, Mahidol University, Bangkok, Thailand
}

\begin{abstract}
Study Design: Prospective, randomized, controlled study.
Purpose: To determine whether the use of goal-directed fluid therapy (GDT) guided by pulse-pressure variation (PPV) and fluid management protocol can reduce intraoperative hypotension, blood transfusion requirements, and postoperative complications in adults undergoing complex spine surgery.

Overview of Literature: Complex spine surgeries involve a significant risk of blood loss and intraoperative hypotension. Previous studies showed that GDT reduces intraoperative hypotension and postoperative complications in these surgery types; however, limited information exists about GDT guided by PPV.

Methods: Sixty adults (18-70 years) patients undergoing complex spine surgeries at Siriraj Hospital, Mahidol University, Thailand were enrolled. Patients were allocated to two groups (30 patients in each) using computer-generated randomization. Intraoperative fluid and vasopressor were administrated via either GDT or standard care. The GDT algorithm used PPV and fluid protocol as the primary tool to guide hemodynamic management. The incidences and episodes of perioperative hypotension were measured as the outcomes.

Results: Fifty-seven patients were analyzed (three patients in the GDT group were excluded). The baseline characteristics and surgical procedures of the two groups did not differ significantly. The prevalence of intraoperative hypotension was $80.0 \%$ for the control group and $66.7 \%$ for the GDT group $(p=0.25)$. Two episodes (1-3) of intraoperative hypotension occurred in the control group, and one episode (0-3) occurred in the GDT group; the difference was not significantly different $(p=0.57)$. The intraoperative blood transfusion requirements and postoperative complications were similar in both the groups. In the subgroup analysis, patients with intraoperative hypotension exhibited a higher incidence of postoperative bowel dysfunction.

Conclusions: PPV-guided GDT and fluid protocol, as compared with standard practice, did not show significant advantages with respect to intraoperative hypotension, blood transfusion, or postoperative complications in patients undergoing complex spine surgery in the prone position.
\end{abstract}

Keywords: Goal-directed therapy; Hypotension; Laminectomy; Prone; Fluid therapy

Received Jan 20, 2021; Revised Jan 20, 2021; Accepted Feb 7, 2021

Corresponding author: Manee Raksakietisak

Department of Anesthesiology, Faculty of Medicine, Siriraj Hospital, Mahidol University, Bangkok 10700, Thailand

Tel: +66-81-488-0620, Fax: +66-24113256, E-mail: manee.rak@mahidol.ac.th 


\section{Introduction}

The performance of major spinal surgeries increased significantly during the previous 2 decades because they provide good functional outcomes [1]. Complex spinal surgeries are associated with prolonged procedures, prone position, and blood loss, all of which can contribute to adverse postoperative events [2]. Hypotension and fluctuations in blood pressure during spinal surgery in the prone position under anesthesia are common [3]. Previous studies showed that the main cause of hypotension in the prone position during lumbar spinal surgery is an inadequate preload, leading to a decrease in the stroke volume (SV) and cardiac index [4]. Furthermore, decreased pulmonary compliance and a rigid supportive spine frame contribute to a decrease in the SV [5]. Elevated risks of end-organ injury owing to intraoperative hypotension have been reported [6]. A fall in splanchnic perfusion results in neurological injury and an intramucosal acidosis of the gut [7], leading to a cascade of events that impair the postoperative neurological outcome as well as gastrointestinal and renal functions. Careful management of fluid and blood pressure during surgery may improve patient outcomes.

The first goal-directed therapy (GDT) was employed by Shoemaker et al. [8] in 1988; they used a pulmonary artery catheter to guide oxygen delivery perioperatively. However, for subsequent GDT studies, less invasive monitoring systems have been used to measure intraoperative SV and cardiac output to guide fluid therapy [9]. The results of randomized, controlled trials and meta-analyses suggest that intraoperative GDT is associated with improved outcomes and reductions in hospital stays for patients undergoing cardiac, major abdominal, and major orthopedic surgeries [10,11]. Next, pulse-pressure variation (PPV) is based on heart-lung interactions and cyclic changes in the SV induced by mechanical ventilation in the closed-chest condition [12]. PPV monitoring has been used for predicting fluid responsiveness. This method is not as invasive as other methods and is not operator dependent; further, it has been validated for use in patients in the prone position [13].

We hypothesized that PPV monitoring coupled with a treatment algorithm could decrease the incidences of intraoperative hypotension, intraoperative blood transfusion, and perioperative complications in patients undergoing elective complex spine surgery in the prone position as compared to standard hemodynamic management.

\section{Materials and Methods}

\section{Patients}

This study was designed as a single center, prospective, randomized trial with two parallel treatment groups receiving either GDT protocols (GDT group) or standard care (control group). It was conducted at Siriraj Hospital, Mahidol University, Thailand from April 2017 to December 2018 after approval was obtained from the Siriraj Ethical Committee (Si826/2016); the project was registered at Clinical Trials.gov (NCT03164811). Sixty patients were randomized into the two study arms. The randomization was performed in blocks of four using computergenerated numbers. The sequence numbers and groups were placed inside concealed envelopes that were opened after obtaining written consent. The researchers enrolled the participants but did not take part in patient care and assessment.

We enrolled all patients who met the inclusion criteria and were willing to participate, as indicated by the provision of written, informed consent. We included low-risk patients (aged 18-70 years, with an American Society of Anesthesiologists [ASA] physical status of 1-3) undergoing elective thoracolumbar spine surgery with instrumentation (mostly pedicle screws and rods), a laminectomy involving three or more levels without instrumentation, or scoliosis surgery. The experience of the spine surgeons varied from 5 to 25 years. The surgeons were blinded. The exclusion criteria comprised renal impairment, severe cardiovascular disease, a body mass index (BMI) of $>30 \mathrm{~kg} /$ $\mathrm{m}^{2}$, complete cord lesion, hemodynamic instability, and respiratory system compliance (Crs) of $<30 \mathrm{~mL} / \mathrm{cmH}_{2} \mathrm{O}$. We excluded patients with cardiac arrhythmia, obesity, or a Crs of $<30 \mathrm{~mL} / \mathrm{cmH}_{2} \mathrm{O}$ because the PPV cut-off points would be different for those groups (Supplement 1).

Patients in the GDT group received a preoperative oral carbohydrate drink ( $400 \mathrm{~mL}$ of $12.5 \%$ glucose solution) between $6 \mathrm{PM}$ and bedtime on the day before the surgery and another $200 \mathrm{~mL}$ on the morning of the day of the surgery. Basal crystalloid infusion was started at $100 \mathrm{~mL} / \mathrm{hr}$ at $7 \mathrm{AM}$ on the day of the surgery. For the patients in the control group, our standard fasting protocol was followed (nothing by mouth after midnight), and standard fluid management was applied in the morning. All the patients 
received general anesthesia with intravenous induction, with propofol (1.5-2 mg/kg), fentanyl (1-2 mcg/ $\mathrm{kg}$ ), and cisatracurium $1(0.12-0.15 \mathrm{mg} / \mathrm{kg})$ being administered to facilitate orotracheal intubation. The anesthesia was maintained with a balanced inhalational technique with desflurane and nitrous oxide in oxygen to keep 1.0-1.5 end-tidal minimum alveolar concentration and infusions of fentanyl $0.5-1.0 \mathrm{mcg} / \mathrm{kg} / \mathrm{hr}$ and cisatracurium $0.06-0.1 \mathrm{mg} / \mathrm{kg} / \mathrm{hr}$. An arterial line was inserted into the radial artery for invasive arterial pressure and PPV by using a Nihon Kohden-BSM 3562 monitor (Nihon Kohden, Shinjuku, Japan) in all the patients in the GDT group and in some patients in the control group (based on the in-charge anesthesiologists). Patients in both the groups were ventilated in a volume-controlled mode using a tidal volume of $8-10 \mathrm{~mL} / \mathrm{kg}$, positive end expiratory pressure of $5 \mathrm{cmH}_{2} \mathrm{O}$, and with a $10 \%$ inspiratory pause to obtain the plateau pressure in order to calculate the Crs in the supine and prone positions. Then, the ventilation rate was adjusted to maintain an end-tidal carbon dioxide pressure 30-40 mm Hg. A urinary catheter was placed for urinary output monitoring. All the patients received intravenous dexamethasone $(10 \mathrm{mg})$ and ondansetron $(8 \mathrm{mg})$ to prevent postoperative nausea vomiting. In order to avoid any interference with intraoperative hypotension and the postoperative renal function, non-steroidal anti-inflammatory drugs, magnesium, and lidocaine were not used. Intraoperative maintenance of mean arterial pressure at $\pm 25 \%$ from baseline was requested for all the patients. This was achieved as per the attending anesthesiologists in the control group and the GDT protocol in the GDT group (Fig. 1, Supplement 1). Once they fulfilled the standard clinical criteria, the patients were extubated either in the operating room or postoperatively.

\section{Outcome measures}

Demographic data (sex, age, BMI, ASA status, underlying diseases, and baseline preoperative investigations) and intraoperative data (number of spinal surgical levels, duration of the surgery, fluid management, intraoperative hypotensive episodes, and the use of vasopressors) were collected for all the patients. Hematocrit and serum creatinine levels were rechecked on the morning of the first postoperative day and, if necessary, were subsequently repeatedly checked. Patients were monitored daily for signs of postoperative complications for 14 days or until their hospital discharge. The primary outcome was the incidence of hypotensive episodes during complex spine surgery, defined as systolic blood pressure $<90 \mathrm{~mm} \mathrm{Hg}$ or $<25 \%$ of the baseline value or need for vasopressor treatment [14]. The secondary outcomes were the incidences of blood transfusions and other complications from inappropriate intraoperative fluid management (Supplement 1).

We conducted sensitivity analyses on (1) the incidences of hypotensive episodes after induction and after positioning and (2) subgroup of patients who underwent more invasive surgery. With an exploratory intent, postoperative complications were compared between patients who experienced intraoperative hypotension and those who did not experience intraoperative hypotension.

\section{Statistical analyses}

The sample size calculation was based on the research of Picard et al. [15]. They found that the incidences of hypotensive episodes were $7 \pm 2$ for the control group and $3 \pm 1.5$ for the GDT group. Using a two independent means for-

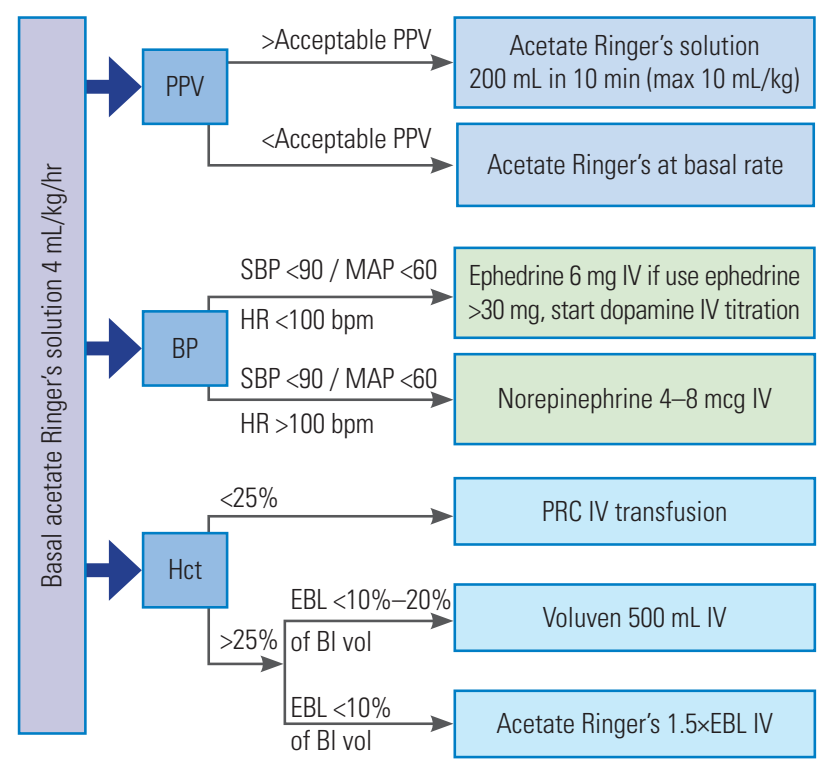

Fig. 1. Goal-directed fluid therapy protocol. Acceptable PPV $=13+\%$ change in static lung compliance after prone position; hypotension: MAP decrease more than $25 \%$ of preoperative MAP value. Preoperative: the day before surgery $(400$ $\mathrm{mL}$ of $12.5 \%$ carbohydrate drinks after 6 PM until the bedtime; on the day of surgery $(200 \mathrm{~mL}$ of glucose solution per oral; acetate Ringer's solution IV rate $100 \mathrm{~mL} / \mathrm{hr}$ since $7 \mathrm{AM}$ ); intraoperative: give acetate Ringer's solution $200 \mathrm{~mL}$ IV within 10 minutes (can repeat up to 3 times or $10 \mathrm{~mL} / \mathrm{kg}$ ) to keep PPV $<13 \%$ before turn to prone position. PPV, pulse-pressure variation; BP, blood pressure; Hct, hematocrit; SBP, systolic blood pressure; MAP, mean arterial pressure; HR, heart rate; EBL, estimated blood loss; BI vol, blood volume; IV, intravenous; PRC, packed red cells. 
mula for the calculation, with a two-sided type 1 error of 0.05 and a power of $90 \%$, two equally sized groups of five patients were needed to detect this difference. Decreasing the incidence of blood transfusions was a secondary outcome. In a study by Bacchin et al. [16], patients received 2 (1-2) units of transfused red blood cells. Assuming a clinically relevant 1-unit reduction in red cell transfusions for the GDT group, with a two-sided type 1 error of 0.05 and a power of $95 \%$, two equally sized groups of 27 patients were needed to detect this difference. Thus, we enrolled 30 patients in each group to compensate for $10 \%$ drop out during the study period. Continuous variables with normal and abnormal distribution were compared using Student $t$ test or Mann-Whitney $U$-test, and the results are presented as mean \pm standard deviation or median and interquartile range (IQR) values, as appropriate. The quality of the discrete variables was compared using Fisher's exact test or chi-square test, and they are presented as numbers and percentages. A value of $p<0.05$ was considered significant. We used statistical software package PASW SPSS Statistics for Windows ver. 18.0 (SPSS Inc., Chicago, IL, USA).

\section{Results}

During the 20-month study period, 170 patients were eligible. However, 110 were excluded; 42 were excluded because they were aged $\geq 70$ years, 33 because they presented with an estimated glomerular filtration rate of $<60$ $\mathrm{mL} / \mathrm{min}, 21$ owing to a history of severe cardiovascular disease, 12 because they presented with a BMI of $>30 \mathrm{~kg} /$ $\mathrm{m}^{2}$, and two because they presented with a complete cord lesion. Sixty patients fulfilled the inclusion criteria (Fig. 2); thus, 30 patients were randomized into each of the two study arms. However, three patients in the GDT group were subsequently withdrawn from the study because of a protocol violation in two cases and a change to the lateral position during the operation in the third case.

No differences were found in the patients' demographic characteristics in terms of age, sex, BMI, ASA physical status, and comorbidities. The preoperative investigations, including the complete blood counts and creatinine levels, were normal and comparable for the two groups (Table 1). Eighty percent of the patients in the control group experienced hypotension compared with $66.6 \%$ of those in the GDT group ( $p=0.25$ ). Most hypotensive episodes occurred after the induction of anesthesia. Sensitivity analysis showed that 16 (53.3\%) patients in the control group and

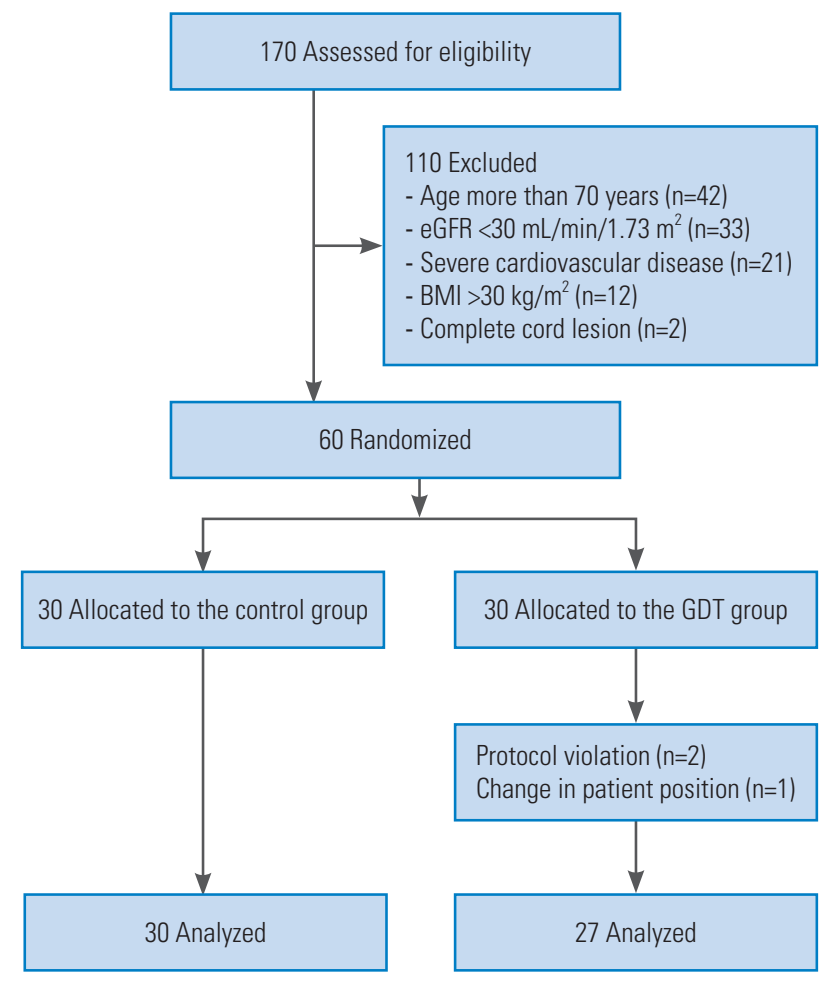

Fig. 2. Consort diagram of the study. eGFR, estimated glomerular filtration rate; $\mathrm{BMI}$, body mass index; GDT, goal-directed therapy.

Table 1. Patient demographic data

\begin{tabular}{|c|c|c|c|}
\hline Characteristic & Control $(n=30)$ & GDT (n=27) & $p$-value \\
\hline Age (yr) & $60.80 \pm 7.78$ & $56.19 \pm 13.02$ & 0.117 \\
\hline Sex & & & 0.153 \\
\hline Male & 11 & 15 & \\
\hline Female & 19 & 12 & \\
\hline Height (cm) & $159.17 \pm 9.07$ & $162.41 \pm 8.91$ & 0.167 \\
\hline Weight (kg) & $60.11 \pm 9.08$ & $65.20 \pm 9.96$ & 0.049 \\
\hline Body mass index $\left(\mathrm{kg} / \mathrm{m}^{2}\right)$ & $23.76 \pm 3.23$ & $24.72 \pm 3.38$ & 0.276 \\
\hline ASA classification 1/2/3 & $10 / 18 / 2$ & $10 / 16 / 1$ & 0.657 \\
\hline \multicolumn{4}{|l|}{ Comorbidity } \\
\hline Diabetes & $6(20.0)$ & $3(11.1)$ & 0.476 \\
\hline Hypertension & $12(40.0)$ & $12(44.4)$ & 0.734 \\
\hline Dyslipidemia & $7(23.3)$ & $8(29.6)$ & 0.590 \\
\hline Preoperative MAP (mm Hg) & $89 \pm 12$ & $94 \pm 10$ & 0.131 \\
\hline Creatinine (mg/dL) & $0.77 \pm 0.17$ & $0.82 \pm 0.23$ & 0.357 \\
\hline $\mathrm{eGFR}\left(\mathrm{mL} / \mathrm{min} / 1.73 \mathrm{~m}^{2}\right)$ & $88.57 \pm 14.48$ & $92.16 \pm 18.28$ & 0.413 \\
\hline Hematocrit (\%) & $39.03 \pm 4.19$ & $40.22 \pm 4.75$ & 0.322 \\
\hline
\end{tabular}

Values are presented as mean \pm standard deviation, number, or number (\%). GDT, goal-directed therapy; ASA, American Society of Anesthesiologists; MAP, mean arterial blood pressure; eGFR, estimated glomerular filtration rate. 
Table 2. Intraoperative data

\begin{tabular}{|c|c|c|c|}
\hline Characteristic & Control $(n=30)$ & GDT (n=27) & $p$-value \\
\hline Type of surgery & & & 0.896 \\
\hline Laminectomy & $1(3.3)$ & $1(3.7)$ & \\
\hline $1-3$ level instrumentation & $15(50.0)$ & $11(40.7)$ & \\
\hline$\geq 4$ level instrumentation & $11(36.7)$ & $11(40.7)$ & \\
\hline Laminectomy with tumor removal & $3(10.0)$ & $4(14.8)$ & \\
\hline Duration of surgery (min) & $236.67 \pm 96.54$ & $220.37 \pm 73.22$ & 0.480 \\
\hline Blood loss (mL) & $575(250-900)$ & $500(325-800)$ & 0.892 \\
\hline \multicolumn{4}{|l|}{ Intraoperative fluid } \\
\hline Crystalloid (mL) & $1,900(1,200-2,800)$ & $2,000(1,425-2,700)$ & 0.904 \\
\hline Colloid (mL) & $0(0-0)$ & $0(0-500)$ & 0.107 \\
\hline Packed red cells (unit) & $0(0-1)$ & $0(0-0)$ & 0.452 \\
\hline \multicolumn{4}{|l|}{ Hypotension } \\
\hline Incidence & $24(80.0)$ & $18(66.6)$ & 0.250 \\
\hline Episodes & $2(1-3)$ & $1(0-3)$ & 0.568 \\
\hline After induction & $16(53.3)$ & $9(33.3)$ & 0.129 \\
\hline After prone & $7(23.3)$ & $4(14.8)$ & 0.416 \\
\hline Medical treatment & $14(46.7)$ & $15(55.6)$ & 0.503 \\
\hline Ephedrine (mg) & $0(0-12)$ & $6(0-9)$ & 0.642 \\
\hline Urine output (mL/kg/hr) & $1.25 \pm 0.76$ & $1.38 \pm 0.99$ & 0.574 \\
\hline Intraoperative oliguric episode & $0(0-1)$ & $0(0-1)$ & 0.424 \\
\hline
\end{tabular}

Values are presented as number (\%), mean \pm standard deviation, or median (interquartile range).

GDT, goal-directed therapy.

$9(33.3 \%)$ in the GDT experienced post-induction hypotension ( $p=0.13$ ); further, 7 (23.3\%) patients in the control group and $4(14.8 \%)$ in the GDT group experienced postpositioning hypotension $(p=0.42)$.

The operations consisted of (1) multilevel laminectomies, (2) laminectomies involving 1-3 levels of instrumentation, (3) laminectomies involving more than three levels of instrumentation, and (4) laminectomies with tumor removal. The four types were equally distributed between the two groups. Thirty-three patients from both the groups exhibited significant blood loss ( $>500 \mathrm{~mL})$. The estimated blood loss and blood transfusion amount of the two groups did not differ (Table 2). The median values of the intraoperative red blood cell transfusions were zero for both the groups, and no differences were found in their intraoperative fluid administration and urine outputs.

On postoperative day 1 , the hematocrit level was $32.0 \% \pm 4.9 \%$ for the control group and $29.9 \% \pm 4.6 \%$ for the GDT group $(p=0.08)$. Although the postoperative complication rate and total length of stay were reduced for the GDT group, the differences were not significant (Table
Table 3. Postoperative data

\begin{tabular}{|c|c|c|c|}
\hline Outcome measure & Control $(n=30)$ & GDT (n=27) & $p$-value \\
\hline Length of hospital stay (day) & $9.5(8.0-15.0)$ & $9.0(7.5-10.5)$ & 0.223 \\
\hline $\begin{array}{l}\text { Unplanned intensive care unit } \\
\text { admission }\end{array}$ & $2(6.7)$ & 0 & 0.492 \\
\hline Renal complications & $18(60)$ & $14(51.9)$ & 0.536 \\
\hline Oliguria (hr) & $2(0-4)$ & $1(0-2)$ & 0.263 \\
\hline Creatinine rising (times) & $0.96(0.86-1.07)$ & $0.96(0.92-1.05)$ & 0.719 \\
\hline Respiratory complication & $2(6.7)$ & 0 & 0.492 \\
\hline Cardiovascular complication & & & 0.708 \\
\hline Episodes of hypotension & $1.5(1.0-4.0)$ & $1.0(0.0-3.0)$ & \\
\hline Cardiac arrhythmia & 0 & 0 & \\
\hline Suspected myocardial infarction & 0 & 0 & \\
\hline Neurological complication & & & 0.490 \\
\hline Stroke & 0 & 0 & \\
\hline Postoperative delirium & $2(6.7)$ & 0 & \\
\hline $\begin{array}{l}\text { New onset of weakness or } \\
\text { numbness }\end{array}$ & 0 & 0 & \\
\hline Bowel ileus & $23(76.7)$ & $16(61.5)$ & 0.219 \\
\hline
\end{tabular}

Values are presented as median (interquartile range) or number (\%). GDT, goal-directed therapy. 
Table 4. Outcome of intraoperative hypotension

\begin{tabular}{lccc} 
& \multicolumn{2}{c}{ Intraoperative hypotension } & \\
\cline { 2 - 3 } Outcome measure & Yes (n=42) & No (n=15) & \\
Length of hospital stay (day) & $9.0(8.0-12.0)$ & $9(7.0-10.0)$ & 0.131 \\
Renal complications & $24(57.1)$ & $8(53.3)$ & 0.517 \\
Postoperative hypotension & $5(11.9)$ & 0 & 0.310 \\
Postoperative delirium & $2(4.8)$ & 0 & 0.539 \\
\hline Stool pass (day) & $4(3.0-5.0)$ & $2(1.0-3.0)$ & $0.005^{*}$ \\
\hline
\end{tabular}

Values are presented as median (interquartile range) or number (\%). Renal complications were defined as oliguria (a urine output of $<0.5 \mathrm{~mL} / \mathrm{kg} / \mathrm{hr} \times 12$ hours) or rising creatinine $\times 2$ compared with baseline.

${ }^{*} p<0.05$; statistically significant.

3). Two patients in the control group continued with mechanical ventilation in the intensive care unit (ICU); one exhibited experienced massive blood loss $(6,000$ $\mathrm{mL})$, while the other received a prolonged operation $(>6$ hours). In the subgroup analysis including more invasive surgical procedure (laminectomies involving more than three levels of instrumentation and laminectomies with tumor removal), the results remain robust, except for the postoperative bowel ileus that GDT provided promising result with marginally significant effect $(p=0.058)$ (Supplement 1). From our exploratory analysis, the patients who experienced hypotension seemed to present with more postoperative complications and delayed recovery of bowel function as compared to those without hypotension $(p=0.005)$ (Table 4).

\section{Discussion}

The perioperative maintenance of an adequate intravascular volume status is important for the achievement of intraoperative hemodynamic stability and optimal postoperative outcomes. Both, hypovolemia and hypervolemia decrease tissue perfusion and may result in organ failure $[17,18]$. GDT is a method of optimizing perioperative fluid administration and reducing the incidence of postoperative organ dysfunction. With regard to spine surgery, Picard et al. [15] conducted a randomized, controlled trial using an esophageal Doppler as a hemodynamic guidance during surgery. Their study showed that the durations and episodes of hypotension were greater in the standard care group than those in the GDT group. In contrast to the findings of the Picard study, the observed effect of the application of the PPV-guided GDT used in our protocol did not significantly reduce the incidences of intraoperative hypotension, despite similar clinical characteristics to those in the Picard study. We reported only 1-2 episodes of hypotension per patient, compared with 3-7 episodes in the Picard study. There could have been several factors that contributed to the differences; for instance, we used desflurane to maintain anesthesia, while Picard et al. [15] used propofol target-controlled infusion. Several studies reported fewer episodes of intraoperative hypotension of inhalation anesthesia with desflurane or sevoflurane than that with propofol $[19,20]$. This study reported a blood loss of about $500 \mathrm{~mL}$; however, our intraoperative transfusion rate was very low (median, 0 ; IQR, $0-1$ ). We used the transfusion guideline obtained from the clinical evidences and guidelines for red blood cell transfusions [14]. These guidelines exhibit a common theme as follows: avoid unnecessary transfusions. In comparison, Bacchin et al. [16] retrospectively reviewed the outcomes in 23 patients who received spine surgery and whose fluid administration had been managed with the GDT protocol as compared to that in 23 matched controls who received a liberal fluid therapy. They found that the application of GDT based on SV variation can lead to a significant reduction in blood loss (median [IQR] from $250[0-500] \mathrm{mL}$ to $0[0-250] \mathrm{mL}$ ) with a reduction of 1 unit of transfused red blood cells, better postoperative respiratory performance, a shorter ICU stay, and a faster normalization of bowel function [16]. However, in this retrospective study, no criteria were present for blood transfusions leading to over-transfusion despite limited blood loss. Few studies reported on goaldirected fluid therapy in spine surgery. Our study results did not reveal any significant benefits of the GDT protocol in comparison to that of standard treatment. Many factors might contribute to this finding. First, the patients in the present study received the same amount of fluid whether they were or were not subjected to PPV monitoring. During the previous decade, the concept of perioperative fluid therapy changed drastically, resulting in the overall intraoperative fluid administration decreasing to the minimum necessary amount [21]. This concept may have helped to lower the incidence of complications and morbidity and may have reduced the marginal benefits of the alternative GDT strategy. Moreover, the many surgical devices and techniques that have been introduced to this field of surgery in recent years contributed toward a reduction in morbidity and mortality $[22,23]$. Finally, the GDT protocol may be more useful in the intraoperative care of high- 
risk patients. Thus far, no definitive data exist to support this belief. Further trials are necessary before a definite conclusion can be made.

Many subsequent trials of the GDT protocol revealed results different from those of the original trial. The GDT for early sepsis patients is a good example. The first randomized controlled trial of GDT for early sepsis cases was performed by Rivers et al. [24] in 2001. More than a decade after their trial, three large multicenter trials investigated the validity of early GDT for managing early sepsis. The North American Protocol-Based Care for Early Septic Shock trial, the UK Protocolised Management in Sepsis (ProMISe) trial, and the Australasian Resuscitation in Sepsis Evaluation trial could not demonstrate a significant survival benefit for protocol-based early GDT over usual care at 90 days [25-27]. A meta-analysis of intraoperative goal-directed fluid therapy for elective major abdominal surgery also indicated that the benefits of GDT may not be as clear as had been suggested historically owing to the changes in the overall perioperative management of patients over time [28]. Based on an exploratory analysis, hypotensive patients experienced delayed bowel function. A decrease in the splanchnic blood flow during a hypotensive episode may lead to mucosal hypoxia and acidosis [7]. Experimental studies showed that the gastrointestinal mucosal $\mathrm{pH}$ decreases with reduction in blood flow [29]. In a clinical setting, postoperative ileus after surgical procedures may contribute to a delay in enteral nutrition, causing patient discomfort and prolonging hospitalization [30]. Certain limitations of this study are present. First, owing to the small number of episodes of hypotension than that reported previously, our sample size might have been too small to demonstrate differences between the two groups. Furthermore, our study only included lowrisk patients; the benefits of GDT for those with a higher risk and severe comorbidities need to be evaluated. Finally, the intervention could not be blinded; however, the risk of bias was minimized by randomization; validated criteria for the primary outcome that was not subjective to observer bias; and the postoperative caregivers being unaware of the study assignment.

\section{Conclusions}

As compared to standard practice, PPV-guided GDT did not show significant advantages with respect to intraoperative hypotension, red blood cell transfusion, or postop- erative complications during complex spine surgery.

\section{Conflict of Interest}

No potential conflict of interest relevant to this article was reported.

\section{Acknowledgments}

The authors would like to thank Mr. Suthipol Udompunthurak for statistical analysis and Miss Nichapat Thongkaew and Miss Chusana Rungjindamai for coordinating this study.

\section{Funding}

This research project was supported by the Faculty of Medicine, Siriraj Hospital, Mahidol University (grant no., R016031008).

\section{Author Contributions}

KW, SW, and MR conceptualized the paper. KW and $\mathrm{NH}$ corrected all the preoperative and intraoperative data. SW and ST followed up the patients and corrected postoperative outcomes. NH analyzed the data, with input from KW, SW, and MR. KW wrote the initial draft with all authors providing critical feedback and edits to subsequent revisions. All authors approved the final draft of the manuscript. MR is the guarantor. The corresponding author attests that all listed authors meet authorship criteria and that no others meeting the criteria have been omitted.

\section{Supplementary Materials}

Supplementary materials can be available from https:// doi.org/10.31616/asj.2020.0597. Supplement 1. Supplementary materials for the manuscript.

\section{References}

1. Marquez-Lara A, Nandyala SV, Fineberg SJ, Singh K. Current trends in demographics, practice, and in-hospital outcomes in cervical spine surgery: a national database analysis between 2002 and 2011. Spine (Phila Pa 1976) 2014;39:476-81.

2. Lamperti M, Tufegdzic B, Avitsian R. Management 
of complex spine surgery. Curr Opin Anaesthesiol 2017;30:551-6.

3. Dharmavaram S, Jellish WS, Nockels RP, et al. Effect of prone positioning systems on hemodynamic and cardiac function during lumbar spine surgery: an echocardiographic study. Spine (Phila Pa 1976) 2006;31:1388-93.

4. Lee TC, Yang LC, Chen HJ. Effect of patient position and hypotensive anesthesia on inferior vena caval pressure. Spine (Phila Pa 1976) 1998;23:941-7.

5. Yokoyama M, Ueda W, Hirakawa M, Yamamoto $H$. Hemodynamic effect of the prone position during anesthesia. Acta Anaesthesiol Scand 1991;35:741-4.

6. Wesselink EM, Kappen TH, Torn HM, Slooter AJC, van Klei WA. Intraoperative hypotension and the risk of postoperative adverse outcomes: a systematic review. Br J Anaesth 2018;121:706-21.

7. Holland J, Carey M, Hughes N, et al. Intraoperative splanchnic hypoperfusion, increased intestinal permeability, down-regulation of monocyte class II major histocompatibility complex expression, exaggerated acute phase response, and sepsis. Am J Surg 2005;190:393-400.

8. Shoemaker WC, Appel PL, Kram HB, Waxman K, Lee TS. Prospective trial of supranormal values of survivors as therapeutic goals in high-risk surgical patients. Chest 1988;94:1176-86.

9. Funk DJ, Moretti EW, Gan TJ. Minimally invasive cardiac output monitoring in the perioperative setting. Anesth Analg 2009;108:887-97.

10. Pearse R, Dawson D, Fawcett J, Rhodes A, Grounds RM, Bennett ED. Early goal-directed therapy after major surgery reduces complications and duration of hospital stay: a randomised, controlled trial [ISRCTN38797445]. Crit Care 2005;9:R687-93.

11. Kapoor PM, Magoon R, Rawat RS, et al. Goal-directed therapy improves the outcome of high-risk cardiac patients undergoing off-pump coronary artery bypass. Ann Card Anaesth 2017;20:83-9.

12. Marik PE, Cavallazzi R, Vasu T, Hirani A. Dynamic changes in arterial waveform derived variables and fluid responsiveness in mechanically ventilated patients: a systematic review of the literature. Crit Care Med 2009;37:2642-7.

13. Yang SY, Shim JK, Song Y, Seo SJ, Kwak YL. Validation of pulse pressure variation and corrected flow time as predictors of fluid responsiveness in patients in the prone position. Br J Anaesth 2013;110:713-20.

14. Carson JL, Guyatt G, Heddle NM, et al. Clinical practice guidelines from the AABB: red blood cell transfusion thresholds and storage. JAMA 2016;316:202535.

15. Picard J, Bedague D, Bouzat P, et al. Oesophageal Doppler to optimize intraoperative haemodynamics during prone position: a randomized controlled trial. Anaesth Crit Care Pain Med 2016;35:255-60.

16. Bacchin MR, Ceria CM, Giannone S, et al. Goal-directed fluid therapy based on stroke volume variation in patients undergoing major spine surgery in the prone position: a cohort study. Spine (Phila Pa 1976) 2016;41:E1131-7.

17. Holte K, Sharrock NE, Kehlet H. Pathophysiology and clinical implications of perioperative fluid excess. Br J Anaesth 2002;89:622-32.

18. Grocott MP, Mythen MG, Gan TJ. Perioperative fluid management and clinical outcomes in adults. Anesth Analg 2005;100:1093-106.

19. Kapoor MC, Vakamudi M. Desflurane: revisited. J Anaesthesiol Clin Pharmacol 2012;28:92-100.

20. Bharti N, Chari P, Kumar P. Effect of sevoflurane versus propofol-based anesthesia on the hemodynamic response and recovery characteristics in patients undergoing microlaryngeal surgery. Saudi J Anaesth 2012;6:380-4.

21. Amaral T, Gambassi M, Sarwahi V, Moguilevtch M, Wollowick A. 213. Intraoperative judicious fluid management and blood conserving techniques in scoliosis surgery. Spine J 2009;9:113S.

22. Rajaee SS, Bae HW, Kanim LE, Delamarter RB. Spinal fusion in the United States: analysis of trends from 1998 to 2008. Spine (Phila Pa 1976) 2012;37:6776.

23. Shillingford JN, Laratta JL, Sarpong NO, et al. Instrumentation complication rates following spine surgery: a report from the Scoliosis Research Society (SRS) morbidity and mortality database. J Spine Surg 2019;5:110-5.

24. Rivers E, Nguyen B, Havstad S, et al. Early goaldirected therapy in the treatment of severe sepsis and septic shock. N Engl J Med 2001;345:1368-77.

25. ARISE Investigators; ANZICS Clinical Trials Group, Peake SL, et al. Goal-directed resuscitation for patients with early septic shock. N Engl J Med 2014;371:1496-506. 
26. ProCESS Investigators, Yealy DM, Kellum JA, et al. A randomized trial of protocol-based care for early septic shock. N Engl J Med 2014;370:1683-93.

27. PRISM Investigators, Rowan KM, Angus DC, et al. Early, goal-directed therapy for septic shock: a patientlevel meta-analysis. N Engl J Med 2017;376:2223-34.

28. Rollins KE, Lobo DN. Intraoperative goal-directed fluid therapy in elective major abdominal surgery: a meta-analysis of randomized controlled trials. Ann Surg 2016;263:465-76.
29. Bass BL, Schweitzer EJ, Harmon JW, Kraimer J. Intraluminal pCO2: a reliable indicator of intestinal ischemia. J Surg Res 1985;39:351-60.

30. Fineberg SJ, Nandyala SV, Kurd MF, et al. Incidence and risk factors for postoperative ileus following anterior, posterior, and circumferential lumbar fusion. Spine J 2014;14:1680-5. 